\title{
CLUSTERS OF GALAXIES IN A FLAT CHDM UNIVERSE
}

\author{
A.HABE, C.HANYU, AND S.YACHI \\ Hokkaido University, Sapporo, Japan
}

\section{Introduction}

Cold and hot dark matter (CHDM) model is one of viable models which can reproduce the large scale structure of the universe. HDM may affect structure of clusters of galaxies in CHDM universe. Bryan et al. (1994) gave numerical results of CHDM model that explain some statistical features of X-ray clusters of galaxies, e.g. X-ray luminosiry-temperature realtion, $L \propto \sim T^{3.5}$, without considering radiative processes. However their numerical resolution is insufficient to resolve the cores of $\mathrm{X}$-ray clusters. So, we simulate the formation of clusters in CHDM universe more carefully.

\section{Models and Numerical Results}

We assume three cosmological models in a flat universe with different mass fraction of dark matter components $\left(\Omega_{\mathrm{HDM}}=0.3,0.2,0, \Omega_{\mathrm{baryon}}=0.05\right)$. Hubble constant is $H_{0}=50 \mathrm{~km} / \mathrm{s} / \mathrm{Mpc}$ and power spectra are normalized by $\sigma_{8 h^{-1}}=\mathbf{0 . 6 6 7}$ for all models. Generating constrained density fluctuation for the initial conditions, we calculate by GRAPE + GRAPESPH code for dark matter motion and hydrodynamics.

From our results, we find that gas density profiles well agree with $\mathrm{X}$-ray observation. We cannot find clear difference among three models on the X-ray luminosity-temperature relation, $L \propto \sim T^{2.0}$, at $z=0$. This result is consistent with the recent results by Choi \& Ryu (1997), and Bryan \& Norman (1997).

\section{References}

Bryan, G.L., Klypin,A. Loken,C., Norman,M.L., and Burns, J.O. : Ap.J., 347, L5 (1994). Bryan, G.L., and Norman,M.L. : preprint, astro-ph/9710107 (1997).

Choi, E., and Ryu, D. : preprint, astro-ph/9710078 (1997). 